\title{
THERMAL PROPERTIES OF UNPARTICLE
}

\author{
SHAO-LONG CHEN \\ Department of Physics and Center for Theoretical Sciences, National Taiwan University, Taipei \\ shaolong@phys.ntu.edu.tw \\ XIAO-GANG HE \\ Department of Physics and Center for Theoretical Sciences, National Taiwan University, Taipei \\ hexg@phys.ntu.edu.tw \\ XUE-PENG HU \\ Department of Physics, Nankai University, Tianjin 300071 \\ YI LIAO \\ Department of Physics, Nankai University, Tianjin 300071 \\ liaoy@nankai.edu.cn
}

\begin{abstract}
We report the study ${ }^{1}$ on the thermal properties of unparticle, a scale invariant sector with a non-trivial infrared fixed point. Unparticle $\mathcal{U}$ with scaling dimension $d_{\mathcal{U}}$ has peculiar thermal properties due to its unique phase space structure. We find that the equation of state parameter $\omega_{\mathcal{U}}$, the ratio of pressure to energy density, is given by $1 /\left(2 d_{\mathcal{U}}+1\right)$ providing a new form of energy in our universe. In an expanding universe, the unparticle energy density $\rho_{\mathcal{U}}(T)$ evolves dramatically differently from that for photons, which makes it possible to have a large unparticle relic density today.
\end{abstract}

Keywords: Unparticle; equation of state.

PACS Nos.: 98.80.Cq, 11.15.Tk, 11.25.Hf, 14.80.-j.

\section{Introduction}

The concept of unparticle, proposed by Georgi in Ref. 2, comes from the observation that certain high energy theory with a nontrivial infrared fixed-point at some scale $\Lambda_{\mathcal{U}}$ may develop a scale-invariant degree of freedom below the scale. Due to its scale invariant feature, unparticle cannot have definite non-zero mass but a continuous mass spectrum and its kinematics is mainly determined by its scaling dimension $d_{\mathcal{U}}$ under scale transformations. The unparticle must interact with normal particles, however feebly, to be physically relevant; and the interaction can be well described in effective field theory (EFT). There has been a burst of studies on various aspects of unparticle physics including cosmological and astrophysical implications. ${ }^{3-10}$ In a glut of unparticle phenomenological studies, either unparticles are treated at 
zero temperature as occurring in ordinary particle physics processes, or the thermal properties of photons are taken for granted and applied to the system of unparticles.

In cosmology the equation of state $(\mathrm{EoS})$ parameter $\omega$, the ratio of pressure $(p)$ to energy density $(\rho)$, for a species of energy carrier, plays a crucial role in determining the properties of the expanding universe. ${ }^{11}$ It determines the energy density at a given temperature since $\rho$ evolves with the Friedmann-Robertson-Walker (FRW) metric scale factor $R$ as $R^{-3(1+\omega)}$. It also fixes the rate of deceleration since the deceleration parameter is proportional to $(1+3 \omega) \rho$. The remarkable progress in precision cosmological measurements in the last or two decades implies that one striking feature of the present composition of the universe in the standard cosmological model is that $95 \%$ of the present universe is dark. It is an important task for modern cosmology to determine various relic energy densities and their EoS parameters. ${ }^{12}$

In this talk we report the investigation ${ }^{1}$ on thermodynamics of unparticles directly from their basic properties. We find that the EoS parameter of unparticle $\omega_{\mathcal{U}}=1 /\left(2 d_{\mathcal{U}}+1\right)$ which is different from $\omega_{D M}=0$ for cold dark matter and $\omega_{\gamma}=1 / 3$ for photon. This demonstrates a novel kind of new energy from unparticle. It might be dubbed unmatter, to be distinguished from CDM and ordinary matter. Then we investigate some of its impacts on cosmology and astrophysics.

\section{Thermal Properties of Unparticle}

When a species of particle $i$ is in thermal equilibrium, its energy and pressure densities $\rho_{i}$ and $p_{i}$ are given by

$$
\begin{aligned}
& \rho_{i}(T)=g_{s} \int E f(\vec{k}) \frac{d^{4} k}{(2 \pi)^{4}} 2 \pi 2 k^{0} \theta\left(k^{0}\right) \delta\left(k^{2}-m_{i}^{2}\right), \\
& p_{i}(T)=g_{s} \int \frac{\vec{k}^{2}}{3 E} f(\vec{k}) \frac{d^{4} k}{(2 \pi)^{4}} 2 \pi 2 k^{0} \theta\left(k^{0}\right) \delta\left(k^{2}-m_{i}^{2}\right),
\end{aligned}
$$

where $E=\sqrt{\vec{k}^{2}+m_{i}^{2}}, g_{s}$ accounts for degrees of freedom like spin and the temperature distribution functions $f(\vec{k})$ are given by

$$
f(\vec{k})=\frac{1}{e^{E / T} \mp 1}
$$

for boson and fermion.

The density of states in four-momentum space is proportional to the $\delta$ function due to the dispersion relation for particles. There is no such a constraint in the case of unparticles, whose density of states is dictated by the scaling dimension $d_{\mathcal{U}}$ of the corresponding field to be proportional to: ${ }^{2}$

$$
\frac{d^{4} k}{(2 \pi)^{4}} \theta\left(k^{0}\right) \theta\left(k^{2}\right)\left(k^{2}\right)^{d_{\mathcal{U}}-2} .
$$


Nevertheless, we can interpret it in terms of a continuous collection of particles with the help of a spectral function $\varrho\left(m^{2}\right) \propto \theta\left(m^{2}\right)\left(m^{2}\right)^{d_{\mathcal{U}}-2}:{ }^{13,14}$

$$
2 \pi 2 k^{0} \theta\left(k^{0}\right) \delta\left(k^{2}-m^{2}\right) \frac{d^{4} k}{(2 \pi)^{4}} \varrho\left(m^{2}\right) d m^{2} .
$$

In this construction, compared to the case of particles of a definite mass, $m^{2}$ serves as a new quantum number to be summed over with the weight $\varrho\left(m^{2}\right)$.

To work out the integrals in Eq. (1), we have to normalize $\varrho\left(m^{2}\right)$. Since unparticles exist only below the scale $\Lambda_{\mathcal{U}}$, the spectrum must terminate there. Beyond the scale, unparticles can be resolved and are no more the suitable degrees of freedom to cope with. This also implies that we should require $T<\Lambda_{\mathcal{U}}$ for self-consistency. We thus find the normalized spectrum,

$$
\varrho\left(m^{2}\right)=\left(d_{\mathcal{U}}-1\right) \Lambda_{\mathcal{U}}^{2\left(1-d_{\mathcal{U}}\right)} \theta\left(m^{2}\right)\left(m^{2}\right)^{d_{\mathcal{U}}-2}
$$

which has the correct limit $\delta\left(m^{2}\right)$ as $d_{\mathcal{U}} \rightarrow 1^{+}$. We assume the gas of unparticle obeys the same distribution as normal particles and therefore obtain the pressure and energy densities for unparticles as follows:

$$
\begin{aligned}
& \rho_{\mathcal{U}}(T)=\left(2 d_{\mathcal{U}}+1\right) g_{s} \frac{\left(\Lambda_{\mathcal{U}}^{2}\right)^{1-d_{\mathcal{U}}}}{4 \pi^{2}} T^{2 d_{\mathcal{U}}+2} \mathcal{C}\left(d_{\mathcal{U}}\right), \\
& p_{\mathcal{U}}(T)=g_{s} \frac{\left(\Lambda_{\mathcal{U}}^{2}\right)^{1-d_{\mathcal{U}}}}{4 \pi^{2}} T^{2 d_{\mathcal{U}}+2} \mathcal{C}\left(d_{\mathcal{U}}\right)
\end{aligned}
$$

with $\mathcal{C}\left(d_{\mathcal{U}}\right)=B\left(3 / 2, d_{\mathcal{U}}\right) \Gamma\left(2 d_{\mathcal{U}}+2\right) \zeta\left(2 d_{\mathcal{U}}+2\right)$ and $\left(1-2^{-(2 d+1)}\right) B\left(3 / 2, d_{\mathcal{U}}\right) \Gamma\left(2 d_{\mathcal{U}}+\right.$ $2) \zeta\left(2 d_{\mathcal{U}}+2\right)$ for bosonic and fermionic unparticles respectively. The above results imply the following EoS parameter for unparticles:

$$
\omega_{\mathcal{U}}=\frac{1}{2 d_{\mathcal{U}}+1}
$$

\section{Cosmological Implications}

Now we find that the EoS parameter for unparticles is very different from that for photons or CDM, and generically lies in between for the dimension $d_{\mathcal{U}}>1$. The ensemble of unparticles thus provides a new form of energy density in our universe, which will have important repercussions for cosmology. We now study their implications in our expanding universe by concentrating on their contribution to the energy density in the universe.

The unparticle energy density at present is determined by its initial value at the decoupling temperature $T_{\mathrm{D}}$ where unparticles drop out of the thermal equilibrium with standard model (SM) particles, and its evolution thereafter which is closely related to the EoS parameter. 
In an FRW expanding universe, the energy densities $\rho(T)$ of photon and unparticle after decoupling from equilibrium are given by

$$
\begin{aligned}
& \rho_{\mathcal{U}}\left(T_{\gamma}\right)=\rho_{\mathcal{U}}\left(T_{\mathrm{D}}\right)\left(\frac{T_{\gamma}}{T_{\mathrm{D}}}\right)^{3(1+\omega \mathcal{U})}, \\
& \rho_{\gamma}\left(T_{\gamma}\right)=\rho_{\gamma}\left(T_{\mathrm{D}}\right)\left(\frac{T_{\gamma}}{T_{\mathrm{D}}}\right)^{4},
\end{aligned}
$$

where $T_{\gamma}$ are the temperature of photons. For $d_{\mathcal{U}}>1$, the unparticle energy density decreases more slowly than the photon's as the universe cools down. A dramatic consequence of this is that even if the unparticle density is small compared with photon density at a high temperature $T_{\mathrm{D}}$, it may become larger or even comparable to the critical density at a lower temperature. This opens the possibility for unparticle to play an important role as a dark composition in the present universe. It provides gravitational attraction, but with EoS parameter deviating from that for cold dark matter. It would be interesting to see whether such a picture fits in a global analysis of various cosmological data.

The decoupling temperature $T_{\mathrm{D}}$ depends on unparticle-particle interactions. In EFT below the scale $\Lambda_{\mathcal{U}}$, there could be many possible interactions between unparticles and SM particles even if unparticles are singlets under the SM gauge group. ${ }^{15}$ For the purpose of illustration, we consider below the unparticle-photon interactions:

$$
\mathcal{L}=\frac{\lambda}{\Lambda_{\mathcal{U}}^{d_{\mathcal{U}}}} F^{\mu \nu} F_{\mu \nu} \mathcal{U}+\frac{\tilde{\lambda}}{\Lambda_{\mathcal{U}}^{d_{\mathcal{U}}}} \tilde{F}^{\mu \nu} F_{\mu \nu} \mathcal{U},
$$

where $F, \tilde{F}$ are respectively the electromagnetic field tensor and its dual, and the coefficients $\lambda, \tilde{\lambda}$ can be expressed in terms of the standard ones in Ref. 15 . We will treat the two interactions one by one.

The above interactions can bring photons and unparticles into equilibrium. Taking the $\lambda$ term as an example, the interaction rate for $\gamma \gamma \rightarrow \mathcal{U}$ is given by

$$
\Gamma \simeq n_{\gamma} \sigma(s) c=\frac{\zeta(3) A_{d_{\mathcal{U}}}}{8 \pi^{2}} \lambda^{2} T\left(\frac{2 T}{\Lambda_{\mathcal{U}}}\right)^{2 d_{\mathcal{U}}},
$$

where $A_{d_{\mathcal{U}}}=16 \pi^{5 / 2} \Gamma\left(d_{\mathcal{U}}+1 / 2\right) /\left((2 \pi)^{2 d_{\mathcal{U}}} \Gamma\left(d_{\mathcal{U}}-1\right) \Gamma\left(2 d_{\mathcal{U}}\right)\right)$ is a normalization factor, ${ }^{2} n_{\gamma}$ is the photon number density and we have also replaced $s$ by $(2 T)^{2}$.

Comparing this rate with the Hubble parameter $H=1.66 g_{*}^{1 / 2} T^{2} / m_{\mathrm{Pl}}$ in the radiation dominated era one obtains the decoupling temperature,

$$
T_{\mathrm{D}}=\frac{1}{2}\left(\frac{1.66 g_{*}^{1 / 2}}{m_{p l}} \frac{\Lambda_{\mathcal{U}}^{2 d_{\mathcal{U}}}}{\lambda^{2} A_{d_{\mathcal{U}}}} \frac{4 \pi^{2}}{\zeta(3)}\right)^{1 /\left(2 d_{\mathcal{U}}-1\right)} .
$$

Here $g_{*}$ is the total degrees of freedom at the decoupling temperature and $m_{\mathrm{Pl}}=$ $1.22 \times 10^{19} \mathrm{GeV}$ is the Planck mass.

In the literature there are constraints on the coupling $\lambda / \Lambda_{\mathcal{U}}^{d_{\mathcal{U}}}$ from experimental data from astrophysics, ${ }^{3-10}$ radiative positronium decay o-P $\rightarrow \gamma \mathcal{U}^{15}$ and $\mathrm{CERN}$ 
Table 1. Upper bound $\left(\lambda / \Lambda_{\mathcal{U}}^{d_{\mathcal{U}}}\right)_{\max }$ (in units of $\mathrm{GeV}^{-d_{\mathcal{U}}}$ ) and the corresponding $T_{\mathrm{D}}^{\min }$ (in units of $\mathrm{GeV}$ ) for various values for $d_{\mathcal{U}}$. Appropriate $g_{*}$ has been used for the given energy with SM particle and a scalar unparticle.

\begin{tabular}{cccc}
\hline$d_{\mathcal{U}}$ & $4 / 3$ & $5 / 3$ & 2 \\
\hline$\left(\lambda / \Lambda_{\mathcal{U}}^{d_{\mathcal{U}}}\right)_{\max }$ & $1.04 \times 10^{-14}$ & $7.17 \times 10^{-13}$ & $5.11 \times 10^{-11}$ \\
\hline$T_{\mathrm{D}}^{\min }$ & $7.37 \times 10^{6}$ & $2.70 \times 10^{3}$ & 36.8 \\
\hline
\end{tabular}

Table 2. $\Lambda_{\mathcal{U}}$ and $r_{\gamma}(T)=\rho_{\mathcal{U}}(T) / \rho_{\gamma}(T)$ as functions of $\Omega_{\mathcal{U}}\left(T_{\gamma}^{0}\right)$. We have used $\rho_{\mathrm{cr}}\left(T_{\gamma}^{0}\right)=8.0992 h^{2} \times 10^{-47}$ $\mathrm{GeV}^{4}$ and taken the central value for $h=0.73_{-0.03}^{+0.04}[16]$.

\begin{tabular}{|c|c|c|c|}
\hline \multicolumn{4}{|l|}{$d_{\mathcal{U}}=4 / 3$} \\
\hline$\Omega_{\mathcal{U}}\left(T_{\gamma}^{0}\right)$ & 1.0 & 0.161 & 0.01 \\
\hline$\Lambda_{\mathcal{U}}(\mathrm{GeV})$ & - & $7.37 \times 10^{6}$ & $4.78 \times 10^{8}$ \\
\hline$r_{\gamma}\left(T_{\mathrm{D}}^{\min }\right)$ & - & 0.990 & 0.0613 \\
\hline$r_{\gamma}\left(T_{\mathrm{BBN}}\right)$ & - & 62.20 & 3.85 \\
\hline \multicolumn{4}{|l|}{$d_{\mathcal{U}}=5 / 3$} \\
\hline$\Omega_{\mathcal{U}}\left(T_{\gamma}^{0}\right)$ & 1.0 & 0.20 & 0.01 \\
\hline$\Lambda_{\mathcal{U}}(\mathrm{GeV})$ & $1.46 \times 10^{4}$ & $4.87 \times 10^{4}$ & $4.61 \times 10^{5}$ \\
\hline$r_{\gamma}\left(T_{\mathrm{D}}^{\min }\right)$ & 0.248 & $4.97 \times 10^{-2}$ & $2.48 \times 10^{-3}$ \\
\hline$r_{\gamma}\left(T_{\mathrm{BBN}}\right)$ & 23.5 & 4.73 & 0.236 \\
\hline \multicolumn{4}{|l|}{$d_{\mathcal{U}}=2$} \\
\hline$\Omega_{\mathcal{U}}\left(T_{\gamma}^{0}\right)$ & 1.0 & 0.2 & 0.01 \\
\hline$\Lambda_{\mathcal{U}}(\mathrm{GeV})$ & $4.31 \times 10^{2}$ & $9.65 \times 10^{2}$ & $4.32 \times 10^{3}$ \\
\hline$r_{\gamma}\left(T_{\mathrm{D}}^{\min }\right)$ & $4.57 \times 10^{-2}$ & $9.11 \times 10^{-3}$ & $4.55 \times 10^{-4}$ \\
\hline$r_{\gamma}\left(T_{\mathrm{BBN}}\right)$ & 3.06 & 0.614 & 0.0305 \\
\hline
\end{tabular}

LEP $e^{-} e^{+} \rightarrow \gamma \mathcal{U} \cdot{ }^{15}$ Taking the most stringent constraints obtained in Ref. 10 we can calculate the allowed maximal coupling $\left(\lambda / \Lambda_{\mathcal{U}}^{d_{\mathcal{U}}}\right)_{\max }$ and the corresponding minimal decoupling temperature $T_{\mathrm{D}}^{\mathrm{min}}$. The actual decoupling temperature can of course be higher than this minimal value. The results are listed in Table 1 . It is seen that $T_{\mathrm{D}}^{\mathrm{min}}$ can vary in a big range from as large as $10^{7} \mathrm{GeV}$ to as low as a few $10 \mathrm{GeV}$ depending on the value of $d_{\mathcal{U}}$.

In Table 2, we list the values of $\Lambda_{\mathcal{U}}$ needed to produce unparticle relic energy density at present photon temperature $T_{\gamma}^{0}=2.725 \pm 0.002 \mathrm{~K}^{16}$ for several representative values of $\Omega_{\mathcal{U}}\left(T_{\gamma}^{0}\right)=\rho_{\mathcal{U}}\left(T_{\gamma}^{0}\right) / \rho_{\mathrm{cr}}\left(T_{\gamma}^{0}\right)$ with $T_{\mathrm{D}}$ set to be $T_{\mathrm{D}}^{\min }$. Here $\rho_{\mathrm{cr}}$ is the critical energy density. Also shown are the values of ratio $r_{\gamma}=\rho_{\mathcal{U}} / \rho_{\gamma}$ at $T_{\mathrm{D}}^{\min }$ and at the Big-Bang-Nucleosynthesis $(\mathrm{BBN})$ temperature $T_{\mathrm{BBN}}=1 \mathrm{MeV}$.

For example, at $d=5 / 3$ we see that the present unparticle relic density can easily saturate the critical density and dark matter density. In all cases, $\rho_{\mathcal{U}}\left(T_{\mathrm{D}}\right)$ is smaller (in most cases much smaller) than $\rho_{\gamma}\left(T_{\mathrm{D}}\right)$. Requiring the present relic of unmatter to be less than these densities one obtains conservative lower bounds on $\Lambda_{\mathcal{U}}$ for given $T_{\mathrm{D}}=T_{\mathrm{D}}^{\mathrm{min}}$. For small $d_{\mathcal{U}}$, the scale $\Lambda_{\mathcal{U}}$ is constrained to be very large, making low energy search for unparticle effects difficult. But for large $d_{\mathcal{U}}$ (close 


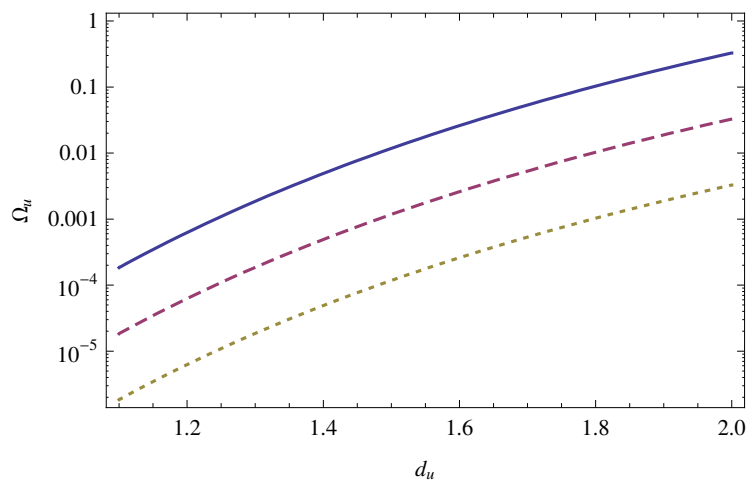

Fig. 1. The relic density of unparticle $\Omega_{\mathcal{U}}$ vs. $d_{\mathcal{U}}$ with assuming the ratio of the energy density of unparticle to photon $r_{\gamma}$ at BBN temperature equals $1,0.1,0.01$ (Solid, Dashed, Dotted) respectively.

to 2), the scale can still be as low as a few hundred GeV which may be reached at LHC and ILC colliders.

The standard BBN theory explains data well. It is therefore important to make sure that at BBN temperature unparticles do not cause problems. A simple criterion is to require that at this temperature the unparticle energy density be less than the photon's. With this restriction, it is interesting to see whether one can still have large relic unmatter at present. We find this is indeed possible. Although there are many cases shown in Table 2 where $r_{\gamma}\left(T_{\mathrm{BBN}}\right)$ is larger than one, circumstances with sizable $\Omega_{\mathcal{U}}\left(T_{\gamma}^{0}\right)$ but small $r_{\gamma}\left(T_{\mathrm{BBN}}\right)$ also appear at large $d_{\mathcal{U}}$. This can easily be understood from the following equation

$$
\Omega_{\mathcal{U}}\left(T_{\gamma}^{0}\right)=\Omega_{\gamma}\left(T_{\gamma}^{0}\right) r_{\gamma}\left(T_{\mathrm{BBN}}\right)\left(\frac{T_{\mathrm{BBN}}}{T_{\gamma}^{0}}\right)^{\frac{2 d_{\mathcal{U}}-2}{2 d_{\mathcal{U}}+1}} .
$$

From Fig. 1, we can see for a fixed ratio at $T_{B B N}$, a larger $d_{\mathcal{U}}$ leads to a richer relic density of unparticle. A universe dominated by unparticle between the BBN era and matter or dark energy dominated universe is possible.

There is much to be explored for the roles that thermal unparticles can play in our universe. It is important to analyze available cosmological and astrophysical data for a global fit with unparticle energy density integrated.

\section{Conclusions}

To recap, we have studied for the first time the thermal properties of unparticles. We found that the equation of state parameter of unparticle $\omega_{\mathcal{U}}$ is given by $1 /\left(2 d_{\mathcal{U}}+1\right)$, which provides a new form of energy in the universe. Therefore in an expanding universe, the behavior of unparticle energy density $\rho_{\mathcal{U}}(T)$ is dramatically different than that for photons. For $d_{\mathcal{U}}>1$, even if $\Omega_{\mathcal{U}}$ is very small at a high decoupling temperature, it is possible to have a large unparticle relic density today. 


\section{Acknowledgments}

The work of authors was supported in part by the grants NSC95-2112-M-002-038MY3, NCTS (NSC96-2119-M-002-001), NCET-06-0211, and NSFC-10775074.

\section{References}

1. S. L. Chen, X. G. He, X. P. Hu, and Y. Liao, arXiv:0710.5129 [hep-ph].

2. H. Georgi, Phys. Rev. Lett. 98, 221601 (2007).

3. H. Davoudiasl, Phys. Rev. Lett. 99, 141301 (2007).

4. S. Hannestad, G. Raffelt, and Y. Y. Y. Wong, Phys. Rev. D 76, 121701 (2007).

5. L. Anchordoqui and H. Goldberg, Phys. Lett. B 659, 345 (2008).

6. J. McDonald, arXiv:0709.2350 [hep-ph].

7. P. K. Das, Phys. Rev. D 76, 123012 (2007).

8. I. Lewis, arXiv:0710.4147 [hep-ph].

9. G. L. Alberghi, A. Y. Kamenshchik, A. Tronconi, G. P. Vacca, and G. Venturi, arXiv:0710.4275 [hep-th].

10. A. Freitas and D. Wyler, JHEP 12, 033 (2007).

11. E. W. Kolb, M. S. Turner, The Early Universe (Westview Press, 1990).

12. U. Seljak, A. Slosar, and P. McDonald, JCAP 0610, 014 (2006).

13. H. Georgi, Phys. Lett. B 650, 275 (2007).

14. K. Cheung, W. Y. Keung, and T. C. Yuan, Phys. Rev. Lett. 99, 051803 (2007).

15. S. L. Chen and X. G. He, Phys. Rev. D 76, 091702 (2007).

16. W.-M. Yao et al., J. Phys. G 33, 1 (2006). 